\title{
COMPARISON OF EXPERIMENTAL RESULTS WITH NUMERICAL SOLUTION OF THERMAL STRESS ANALYSIS
}

This paper presents experimental measurement of the first stress invariant on the plate with hole at fatigue testing machine due to adiabatic elastic deformation. The theoretical part is concentrated on the theory of thermal stress analysis focusing on thermoelastic analysis. The experimental part is dedicated to the postprocessing of the measured data including analytical and numerical solutions for the plate with hole using finite element method (FEM).

Keywords: Thermoelastic, thermal stress analysis, infrared camera, first stress invariant, temperature.

\section{Introduction}

The determination of stress and strain fields can be made in many ways. It can be used, for example, classical strain gauges [1], optical methods based on correlation of the image [2] but also on the base of detection of infrared radiation.

The deformation of structural materials is followed by thermal effects. We recognize thermoelastic or thermoplastic stress analysis, depending on whether the load creates elastic or plastic strains. Thermoelastic stress analysis describes the relation between stress changes and temperature changes of a body in specimens. When the tensile deformation is in the elastic field specimen's temperature increases, on the other hand when there is a pressure load it decreases. The thermoplastic effect quantifies the heat generated by plastic deformation. In the elastic part it is possible under adiabatic conditions to determine the value of the first stress invariant on the material surface by measuring changes of the surface temperature. Adiabatic conditions are ensured by frequency higher than $2 \mathrm{~Hz}$ for steel specimens and more than $20 \mathrm{~Hz}$ for aluminum specimens (aluminum sample was used in experiment by Estrada [3]). In plastic zone it is possible only to estimate the trace of stress tensor, because there is not a total conversion of mechanical energy into heat.

Surface temperature in thermal stress analysis was previously measured using thermocouples. The development of new technologies brought a new contactless method for measuring the temperature with greater sensitivity by using thermography. Infrared thermography is a unique technology which allows using an infrared cooled detector to measure the surface temperature of an object. It is possible to determine the temperature changes on the surface of the measured object from the thermogram [4]. Rajic describes the development and validation of a novel thermoelastic stress analysis system based on a low cost microbolometer device [5].

Most of applications of thermal stress analysis concern FEM comparison, fatigue testing and vibration analysis in automotive industry, naval, aircraft and steel industry [6].

\section{Thermal stress analysis}

Thermoelastic and thermoplastic effect are summarized in a 3 - dimensional heat equation together with the effect of heat conduction [7 and 8]:

$$
\begin{aligned}
& \rho C_{\varepsilon} \frac{\partial T}{\partial t}=k\left(\frac{\partial^{2} T}{\partial x^{2}}+\frac{\partial^{2} T}{\partial y^{2}}+\frac{\partial^{2} T}{\partial z^{2}}\right)+ \\
& +T_{0} \sum \frac{\partial \sigma_{i j}}{\partial T} \varepsilon_{l J}^{\cdot e}+\alpha_{p} \sigma_{i j} \varepsilon_{l j}^{\cdot p}
\end{aligned},
$$

where $\rho$ is the material density, $C_{\varepsilon}$ is specific heat capacity at constant deformation, $T$ is absolute temperature, $t$ is time, $k$ thermal conductivity, $x, y$ and $z$ are spatial coordinates, $T_{0}$ is the initial temperature, $\sigma_{i j}$ stress tensor, $\varepsilon_{I J}{ }^{e}$ is the rate of change of elastic deformation, $\alpha_{p}$ is the ratio of plastic deformation, which is converted to total heat of plastic deformation, $\varepsilon_{I J}^{{ }^{P}}$ is the irreversible part of deformation tensor [7].

\footnotetext{
* ' Zuzana Stankovicova, ${ }^{1}$ Vladimir Dekys, ${ }^{2}$ Leszek Radziszewski, ${ }^{1}$ Milan Uhricik, ${ }^{1}$ Milan Sapieta 


\subsection{Thermoelastic effect}

Thermoelastic effect is known as the conversion between mechanical forms of energy and heat. This transformation occurs when stress changes within a material element alter its volume. Density of energy generated in an object is transformed into local temperature changes. If specific heat of metal is high this phenomenon is insignificant in terms of temperature change. Roughly 1 MPa change in stress state causes a temperature change of $1 \mathrm{mK}$ in steel [6].

The equation of thermoelasticity is derived from heat equation ignoring thermoplastic effect [9]:

$\rho C_{\varepsilon} \frac{\partial T}{\partial t}=k\left(\frac{\partial^{2} T}{\partial x^{2}}+\frac{\partial^{2} T}{\partial y^{2}}+\frac{\partial^{2} T}{\partial z^{2}}\right)+T_{0} \sum \frac{\partial \sigma_{i j}}{\partial T} \varepsilon_{l J}^{\cdot e}$

To evoke the thermoelastic effect it is standard to load object cyclically so that no heat conduction takes place. Therefore the first term on the right side of (2) can be neglected. Time integration of (2) ignoring heat transfer leads to the following equation:

$\rho C_{\varepsilon} \Delta T=T_{0} \sum \frac{\partial \sigma_{i j}}{\partial T} \mathcal{E}_{i j}^{e}$ pre $i, j=1,2,3$.

Using Hooke's law and simple mathematical operations we obtain an expression that relates the trace of stress tensor (first stress invariant) $\sigma_{i i}$ and temperature changes $\Delta T$

$$
\sum_{i=1,2} \sigma_{i i}=-\frac{\Delta T}{\alpha}\left[\frac{\rho C_{\varepsilon}}{T_{0}}-\frac{2 \alpha^{2} T_{0}}{1-v}\right] .
$$

where $\quad v$ - Poisson's ration (-)

$\alpha$ - linear coefficient of thermal expansion $\left(K^{I}\right)$.

Equation (4) can be simplified by using the relationship between heat capacity at constant deformation $C_{\varepsilon}$ and heat capacity at constant pressure $C_{p}[9]$ :

$C_{\varepsilon}=C_{p}-\frac{2 E \alpha^{2} T_{0}}{\rho(1-v)^{\prime}}$

where $E$ - Young's modulus $(\mathrm{Pa})$.

Substituting (5) to (4) we obtain

$\Delta T=-\frac{\alpha}{p C_{p}} T_{0} \sum_{i=1,2} \sigma_{i i}$

The term $\frac{\alpha}{p C_{p}}$ is a proportionality constant known as thermoelastic constant $K$. Therefore (6) can be rewritten as:

$\Delta T=-K T_{0}\left(\sigma_{1}+\sigma_{2}+\sigma_{3}\right)$,

where, $\sigma_{1}, \sigma_{2}, \sigma_{3}$, are changes in the principal stresses. The sum of principal stresses is known as the first invariant of stress or trace of stress tensor. Equation (7) says that the sum of the principal stresses is related to the dilatational component of deformation. Tensile load causes a decrease of temperature and vice versa.

\section{Experimental part}

The experimental part consists of measuring the temperature change by using an infrared camera in elastic field during cyclic tension - compression load. The measurement was carried out at the Department of material engineering, which has a frequency Zwick Roel pulsator. The sample was loaded by dynamic amplitude of $1 \mathrm{kN}$ with a frequency of $104 \mathrm{~Hz}$. The measurement was performed on a plate with a hole. On the specimen a black emissivity spray (for LWIR) was applied to prevent the partial reflection of the surrounding surfaces. The emissivity spray for MWIR was not used, this problem is difficult and complex [10].

\subsection{Measurement preparation}

To measure the temperature change an infrared camera with a cooled detector FLIR SC7000 was used (Fig. 1). Three software were used to evaluate the measured data: Research IR MAX, ALTAIR LI, ALTAIR. The software ALTAIR LI provides thermograms of stress fields using thermoelastic effect, which is based on a linear relationship between the temperature changes induced mechanical load and stress at the surface of the material. ALTAIR LI software is associated with the Lock In method that is used to extract the signal from noise and for synchronization signal load to signal measured data.

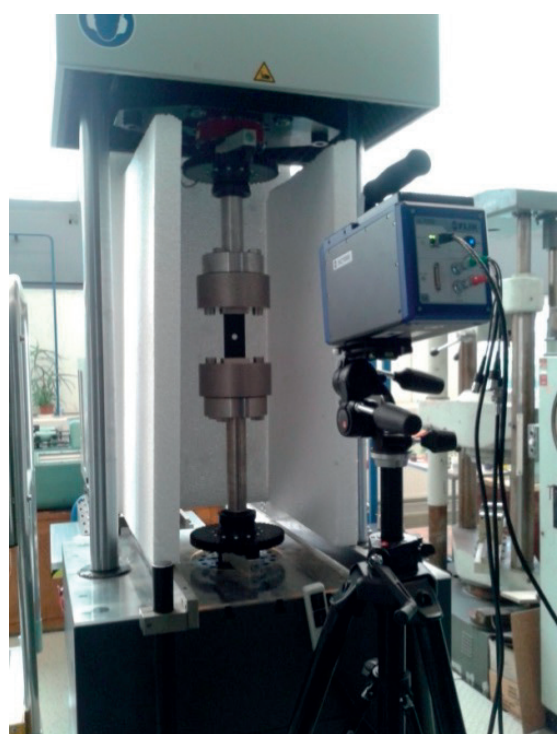

Fig. 1 Measuring the temperature changes using infrared camera FLIR SC7000 and frequency pulsator 
The sample used in the experiment was a steel plate with dimensions $50 \times 111 \mathrm{~mm}$ with a thickness of $1 \mathrm{~mm}$. In the middle of the plate a hole was drilled with a diameter of $12 \mathrm{~mm}$ (Fig. 2). The specimen material is steel $\mathrm{S} 355 \mathrm{~J}$ with properties:

- Young's modulus E=200 GPa,

- Poisson's ratio $\mu=0.3$,

- density $\rho=7800$.

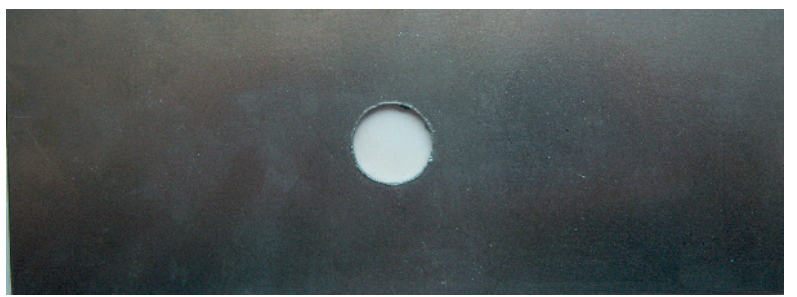

Fig. 2 Steel plate with hole

\subsection{Analytical solution}

In plane stress problems during the applied loading of plate with hole results in stress concentration at the edge of the hole. For an infinite plate the stress is equal to three times the stress in full cross section. For finite plate (cantilevered on one side), the value is slightly higher. The maximum stress in place of stress concentrator is analytically calculated by the stress concentration factor $K_{t}$ :

$\sigma_{\max }=K_{t} \cdot \sigma_{\text {nom }}$.

The stress concentration factor is determined from the graph in Fig. 3. Nominal stress is stress in cross section at half the length of the bar:

$\sigma_{\text {nom }}=\frac{P}{(D-2 r) \cdot \text { thickness }^{\prime}}$

where $P$ is loading force in $N$ and dimensions are given according to Fig. 2.

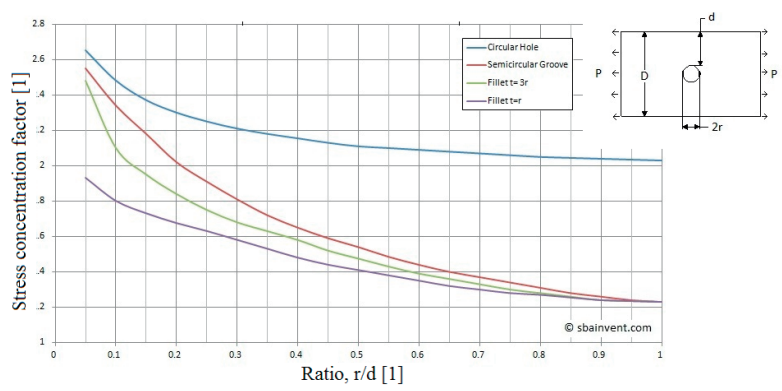

Fig. 3 Stress concentration factor

(http://engineering-references.sbainvent.com/strength_of_materials/ stress-concentrations.php\#.VglAK5cl-VM)
If the sample is loaded with force $1 \mathrm{kN}$, the nominal stress is calculated:

$$
\begin{aligned}
& \sigma_{\text {nom }}=\frac{P}{(H-2 r) \cdot \text { thickness }}= \\
& =\frac{1000}{(50-12) \cdot 1}=26.3 \mathrm{MPa}
\end{aligned}
$$

and the stress in the field of stress concentration:

$\sigma_{\max }=2,425 \cdot 26,3=63.8 \mathrm{MPa}$

In full cross section stress evoked by loading force of $1 \mathrm{kN}$ is $20 \mathrm{MPa}$. In the place of stress concentration it is $64 \mathrm{MPa}$ after rounding.

\section{Measurement results}

Figure 4 shows thermograms of stress fields under tension (Fig. 4a) and compression loading (Fig. 4b). Here we can see that the maximum stress is at the edge of the hole as we assumed in the analytical solution.

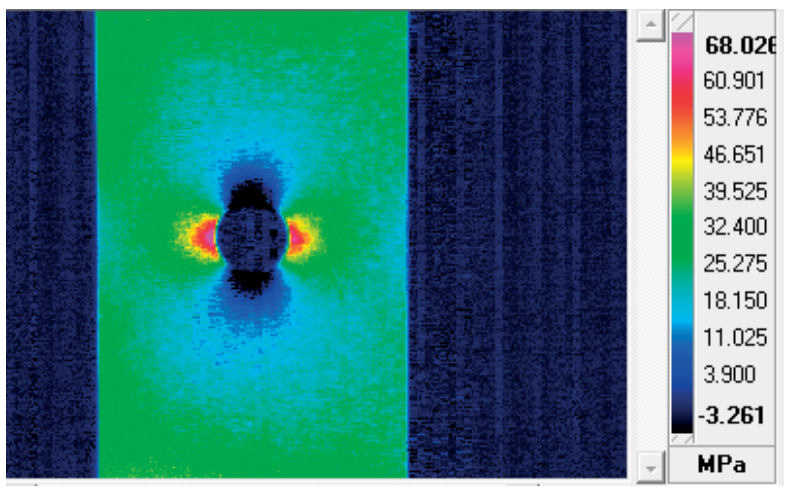

a)

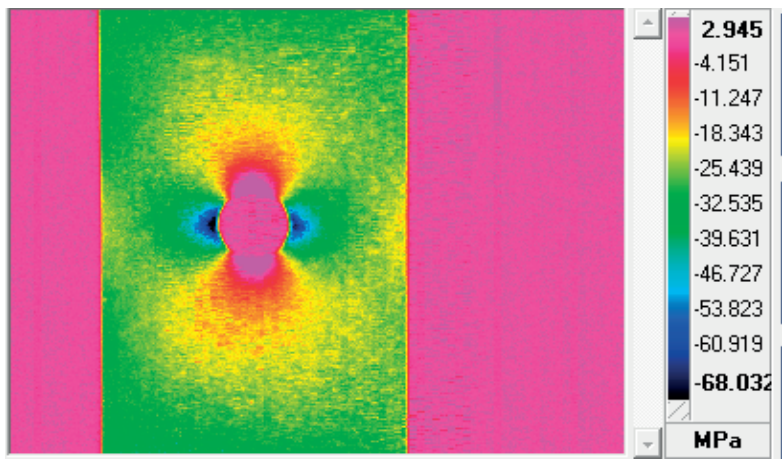

b)

Fig. 4 Measured results for a) tension loading, b) compression loading

Further processing of the results was carried out in ALTAIR software, which provides the user greater comfort with 


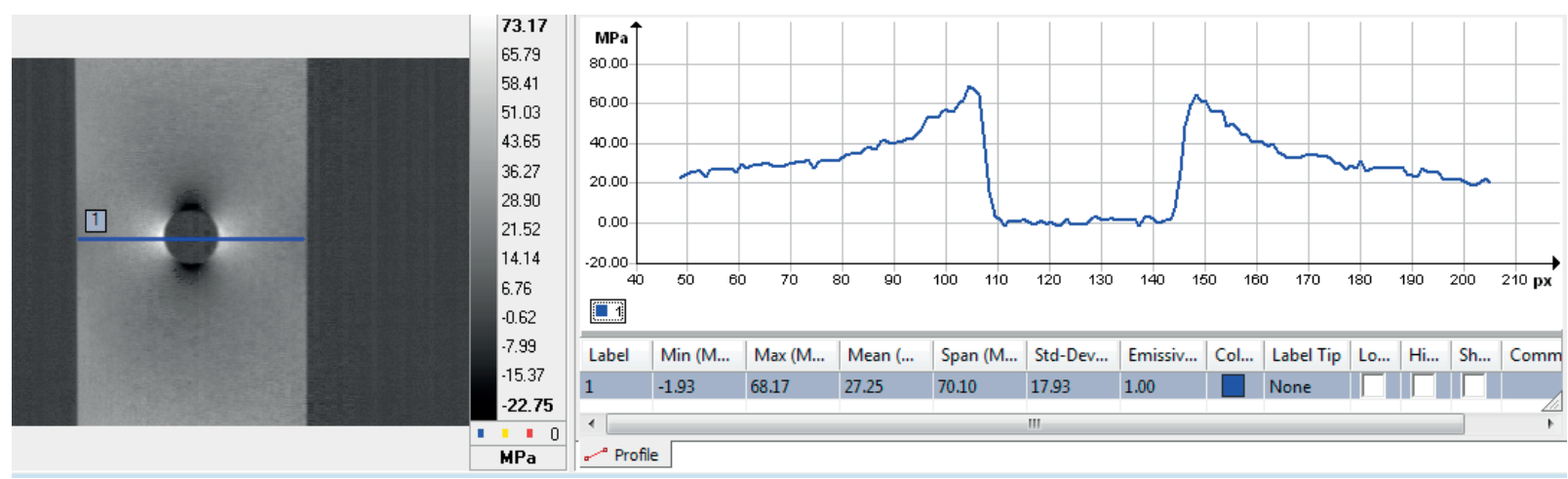

Fig. 5 Measured results for tension loading

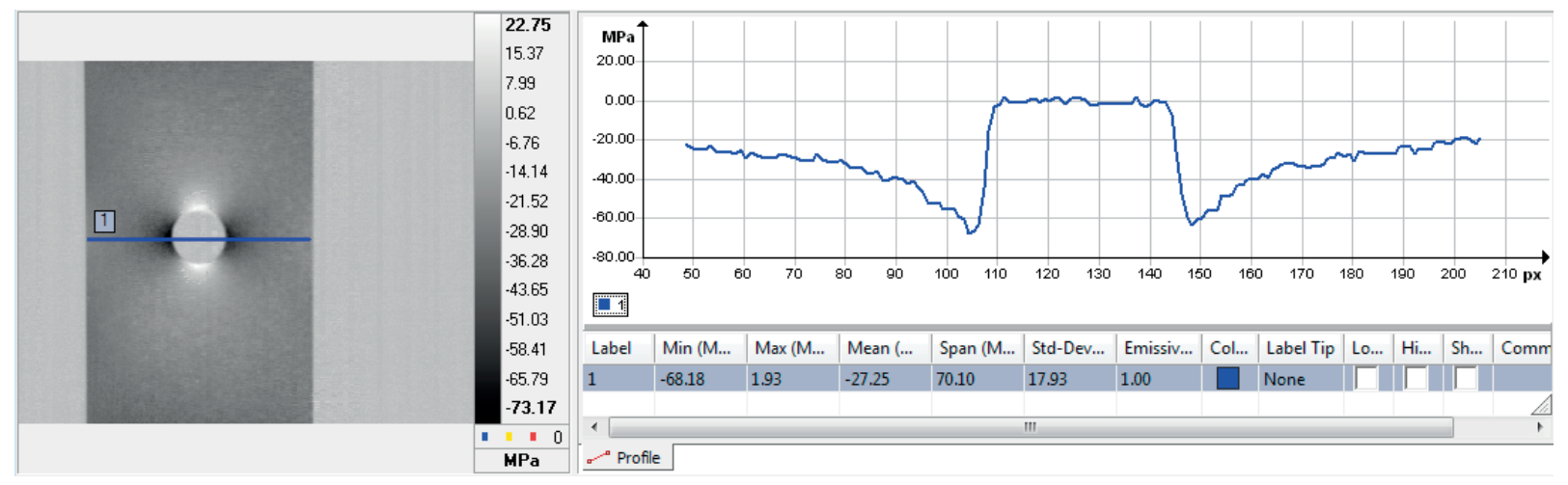

Fig. 6 Measured results for compression loading

processing of measured data. For a sample in tension loading (Fig. 5) and compression loading (Fig. 6) was chosen transverse line and plotted curve of the first stress invariant in each pixel (px). In Fig. 5 you can see that maximum first invariant stress is on the edge of the hole and its value is $68.17 \mathrm{MPa}$. The maximum value during compression loading is -68.18 $\mathrm{MPa}$.

\section{Numerical solutions}

Numerical solution was performed by finite element method in two software: ANSYS and ABAQUS. Structural analyses were created to find out stress field of the specimen during cyclic loading tension - compression. We present results during tension loading. The applied material and boundary conditions were defined according to the experiment. In both of software mesh was generated by 4 node element which is used for modeling plane stress. The result of the structural analysis in software ANSYS for tensile loading is presented in Fig. 7. Here we can see that maximum first stress invariant is 65.2MPa.

Numerical solution performed in software ABAQUS is in Fig. 8. Here we can see that that maximum first invariant stress is on the edge of the hole and its value is $65.3 \mathrm{MPa}$.
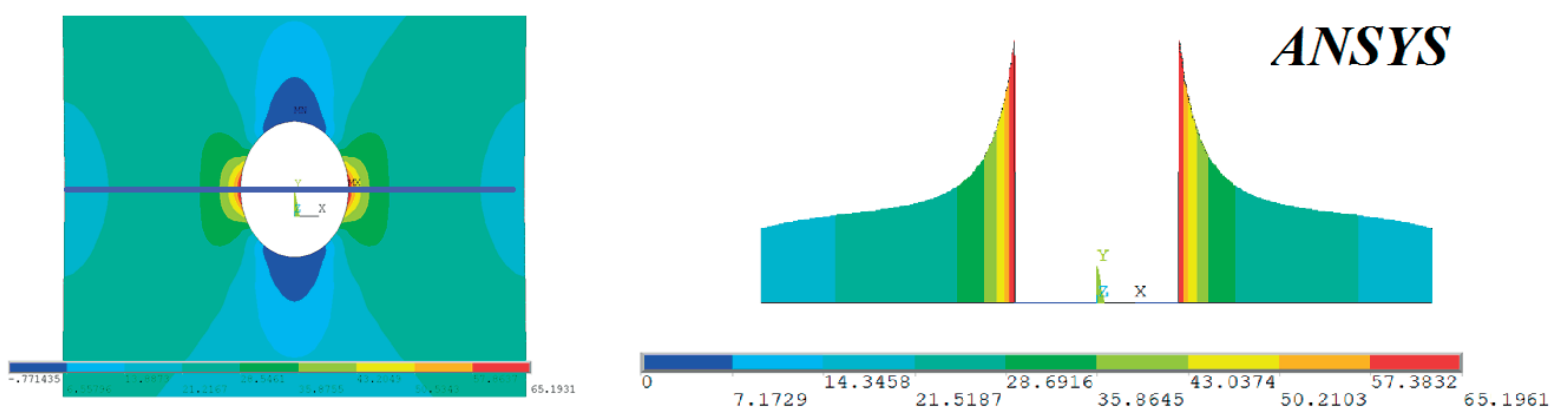

Fig. 7 Numerical solutions for tensile loading in ANSYS software in MPa 


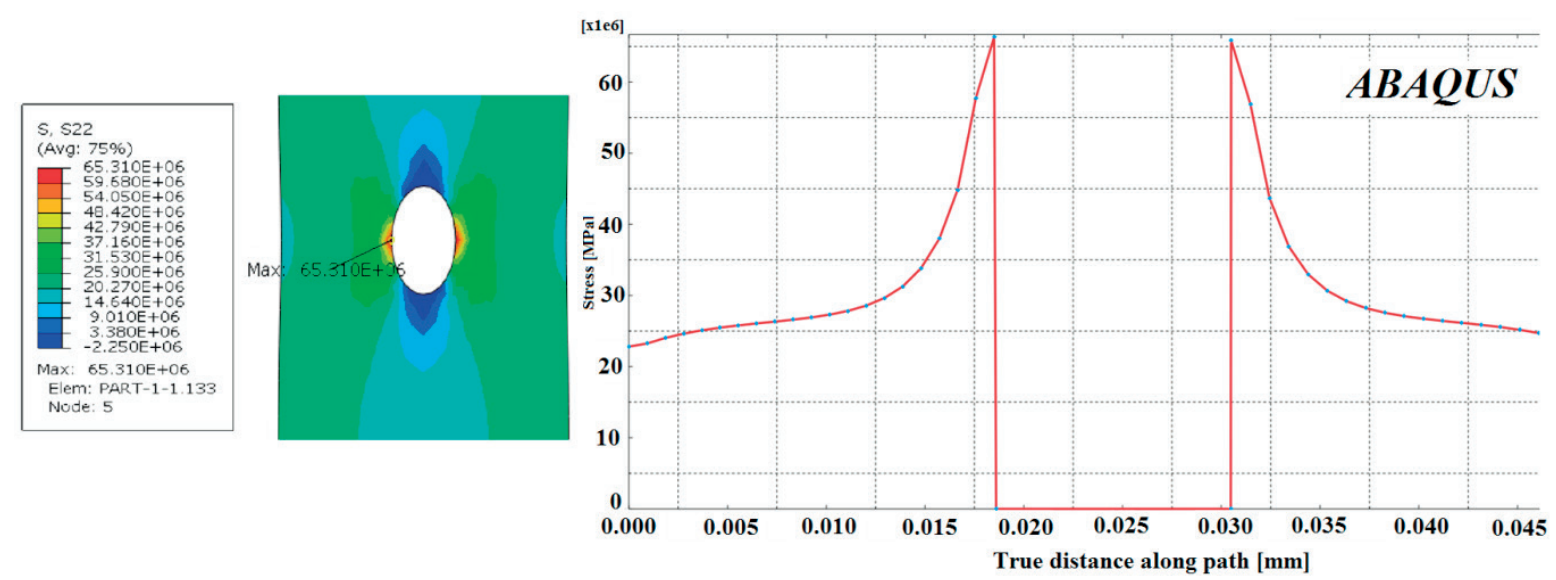

Fig. 8 Numerical solutions for a tensile loading in ABAQUS software in MPa

\begin{tabular}{|c|c|c|c|c|}
\hline & \multirow{2}{*}{ Experiment } & \multirow{2}{*}{ Analytical solution } & \multicolumn{2}{|c|}{ Numerical solution } \\
\hline & & & ANSYS & ABAQUS \\
\hline Maximum first stress invariant in tension loading [MPa] & 68.17 & 63.8 & 65.2 & 65.3 \\
\hline
\end{tabular}

\section{Conclusion}

The paper deals with thermal stress analysis focusing on the thermoelastic analysis. The introduction provides an overview of the theory of thermal stress analysis. The experimental part is preceded by the preparation of the measurement including analytical solution for the plate with a hole. Measured data are compared with numerical solution using the finite element method.

In this paper we compare measured data with analytical and numerical solutions (Table 1). The specimen used for measurement was selected due to better comparison of results. During solution of plane stress of plate with hole stress concentration at the edge of the hole occurs. The error in measurements is less than $5 \%$ compared to numerical solutions under tension loading.

Very interesting area for further measurements based on infrared thermography is determining the stress fields in the composite material [11, 12 and 13].

\section{Acknowledgement}

This work was supported by the Slovak Research and Development Agency under the contract No. APVV-0736-12.

\section{References}

[1] DEKYS, V., BRONCEK, J.: Communications - Scientific Letters of the University of Zilina, 14, 2, 2012.

[2] ZMINDAK, M., NOVAK, P.: Communications - Scientific Letters of the University of Zilina, 14, 4A, 2012, p. 85.

[3] ESTRADA, J. R., PATTERSON, E. A.: Path Dependency in Thermoelastic Stress Analysis. Experimental mechanics, vol. 44, No. 6, 2004, pp. 567-573.

[4] EISENLOHR, A.: Adiabatic Temperature Increase Associated with Deformation Winning and Dislocation Plasticity. [online]. 2012. Available online at www.sciencedirect.com, 2012.

[5] RAJIC, N., ROWLANDS, D.: Thermoelastic Stress Analysis with a Compact Low Cost Microbolometer System. Quantitative Infrared Thermography, vol. 10, No. 2, 2013, pp. 135-158.

[6] BREMOND, P.: New Developments in ThermoElastic Stress Analysis by Infrared Thermography. [online]. 2007. Available online at: < http://www.ndt.net/article/panndt2007/papers/138.pdf >.

[7] PLUM, R. et al.: Extended Thermoelastic Stress Analysis Applied to Carbon Steel and CFRP. [online]. 1999. Available online at: < http://ebookbrowsee.net/we5a3-pdf-d75918406 >.

[8] WANG, G. et al.: Thermographic Studies of Temperature Evolutions in Bulk Metallic Glasses: An overview. Intermetallics. [online]. 2012. Available online at: <www.sciencedirect.com>. ISSN: 0966-9795. 


\section{COMMNICOIIONS}

[9] BARTON, J.: Introduction to Thermoelastic Stress Analysis. Strain [online]. 1999. Available online at: < http://onlinelibrary.wiley. com/doi/10.1111/j.1475-1305.1999.tb01123.x/abstract >. ISSN: 0039-2103

[10] HONNER M., HONNEROVA, P.: Survey of Emissivity Measurement by Radiometric Methods. Applied Optics, vol. 54, 2015, 2015, pp. 669-683. < http://dx.doi.org/10.1364/AO.54.000669>.

[11] ZMINDAK, M., NOVAK, P.: Communications - Scientific Letters of the University of Zilina, 14, 3A, 2012, p. 73.

[12] NOVAK, P., ZMINDAK, M., PELAGIC, Z.: Applied Mechanics and Materials, 486, 2014, p. 181.

[13] RIECKY, D., ZMINDAK, M., PELAGIC, Z.: Communications - Scientific Letters of the University of Zilina, 14, 3A, 2012 , p. 142. 